\title{
HOLONOMY INVARIANT COCHAINS FOR FOLIATIONS
}

\author{
JOHN M. FRANKS ${ }^{1}$
}

\begin{abstract}
The notion of a holonomy invariant cochain for a foliation is developed generalizing the idea of a holonomy invariant measure. An example is given on a foliation in which every leaf has exponential growth, and it is shown that if the inclusion of every leaf is trivial on $H_{1}$ for a codimension one transversally oriented foliation, then holonomy invariant cochains generate all of the one dimensional cohomology.
\end{abstract}

An important recent tool in the study of foliations is the notion of a transversal invariant or holonomy invariant measure (see for example [2], [3], or [4]). In this note we generalize this concept and consider holonomy invariant cochains, i.e. singular cochains defined on chains made up of simplices transverse to the foliation and which assign the same value to two simplices if there is a holonomy transformation carrying one of them to the other. It is almost immediate that such a cochain is a cocycle and hence determines a cohomology class. Transversal invariant measures have most often been thought of as defining homology classes, though consideration of the Poincaré dual cohomology class is implicit in several articles. In a transversely oriented codimension $k$ foliation, a transverse invariant measure immediately gives a real valued holonomy invariant $k$ cochain by assigning to each simplex its measure with a sign depending on whether the orientation of the simplex coincides with that of the foliation (see [3]).

An advantage of considering holonomy invariant cochains is that there are many more of them than invariant measures. For example, J. Plante has shown [3] that codimension one foliations with every leaf of exponential growth have no transverse invariant measures, but we give below an example of such a foliation with a natural holonomy invariant cochain with values in a finite group. Even with real coefficients one gets much more cohomology from invariant cochains than invariant measures. We show below that if the inclusion of each leaf is trivial in one dimensional homology for a transversally oriented codimension one foliation, then the holonomy invariant cochains generate all of the one dimensional cohomology. In fact in this case there is a "universal" holonomy invariant cochain which takes values in $H_{1}(M)$ and assigns to each cycle $z$, the homology class $[z] \in H_{1}(M)$. All homology and cohomology groups will be with integer coefficients unless

Received by the editors April 23, 1976.

AMS (MOS) subject classifications (1970). Primary 57D30.

${ }^{1}$ Supported in part by NSF Grant GP42329X. 
otherwise explicitly noted. All foliations are assumed to be of class $C^{1}$. It is a pleasure to thank R. Bowen for valuable conversations leading to the ideas of this note.

In [5], Thurston showed that any compact manifold with a smooth codimension $k$ foliation admits a smooth triangulation in which all simplices of dimension $\geqslant k$ are transverse to the foliation and that such a triangulation has refinements of arbitrarily small mesh with the same property. Our cochains will be defined only on simplices of such a triangulation. It will sometimes be advantageous to further restrict the class of simplices on which a cochain is defined; for example in a codimension one foliation with a dense leaf $L$ we may allow only transverse one simplices whose vertices lie on $L$.

We will say a class of $k$-simplices is sufficient provided that there exist triangulations of $M$ of arbitrarily small mesh all of whose $k$-simplices are in this class. Clearly to define a cohomology class it is enough that a cochain be defined on a sufficient class of simplices. The theorem of Thurston mentioned above says that the class of smooth $k$-simplices transverse to a codimension $k$ foliation is sufficient.

We recall that a holonomy transformation is a homeomorphism $h$ from one smooth transversal to another with the property that $x$ and $h(x)$ always lie on the same leaf (see [3] for example).

Definition. A holonomy invariant $k$ cochain $c$ for a foliation $\mathscr{F}$ is a cochain defined on a sufficient class of $k$-simplices which are smooth and transverse to $\mathscr{F}$, and which assigns the same value to two simplices if one is the image of the other under a holonomy transformation. We assume as part of the definition of cochain that if $\alpha$ is a singular simplex for which $c$ is defined and it is subdivided into $\alpha=\sum \alpha_{i}$, then if $c$ is defined on each $\alpha_{i}, c(\alpha)=\sum c\left(\alpha_{i}\right)$.

Proposition. A holonomy invariant cochain is a cocycle.

Proof. We want to show that a holonomy invariant cochain $c$ vanishes on chains which are boundaries. It suffices to show it vanishes on the boundary of a transverse $k+1$ simplex $\Delta$ which is sufficiently small that it is contained in a distinguished chart (flow box) for the foliation. We also assume $\Delta$ is so small that the tangent planes to leaves of the foliation are almost constant in a chart in which $\Delta$ is a linear simplex. We choose a transversal $T$ to $\mathscr{F}$ in this chart which is linear and let $X$ be the image of the projection $\pi$ of $\Delta$ along leaves into this transversal. Now $\pi$ is a $C^{1}$ approximation to a linear map and $\pi$ restricted to each face of $\Delta$ is an embedding into the transversal $T$. Thus we can triangulate $X=\pi(\Delta)$ and $\partial \Delta$ so that each $k$-simplex in $X$ is the image under the holonomy transformation $\pi$ of exactly two $k$-simplices of $\partial \Delta$ but with opposite orientations. This fact would be immediate if $\pi$ were linear, but having $\pi$ be a $C^{1}$ perturbation of a linear projection is sufficient (by subdividing and choice of $T$, the map $\pi$ restricted to a simplex can be made arbitrarily close to a linear map in the $C^{1}$ sense). Hence $c(\partial \Delta)=0$, for all sufficiently small transverse $k+1$ simplices and it follows $c$ is a cocycle. 
THEOREM. If $\mathscr{F}$ is a $C^{1}$ transversely oriented codimension one foliation and $K$ is the subgroup of $H_{1}(M)$ generated by $\cup \operatorname{Im}\left(H_{1}(L) \rightarrow H_{1}(M)\right)$ as $L$ varies over all leaves, then there exists a holonomy invariant cochain $c$ with values in $H_{1}(M) / K$ such that if $z$ is any one dimensional cycle in $M$, then $c(z)$ is the coset of $[z]$ in $H_{1}(M) / K$.

COROLlary. If the inclusion $H_{1}(L) \rightarrow H_{1}(M)$ is trivial for each leaf $L$ of a $C^{1}$ codimension one transversely oriented foliation, then holonomy invariant cochains generate $H^{1}(M ; G)$ for any group $G$. Moreover, the cochain $c$ of the Theorem is universal in the sense that if $\bar{c}$ is a holonomy invariant cochain with values in a group $G$, then the cohomology class it determines coincides with the cohomology class of $\psi \circ c$ for some homomorphism $\psi: H_{1}(M ; Z) \rightarrow G$.

Proof of Theorem. Let $I$ be a smoothly embedded arc in $M$ which intersects each leaf of $\mathcal{F}$ and for each leaf $L$ choose one point $x(L)$ in $I \cap L$. We will define the cochain $c$ on all one-simplices which are smoothly embedded and transverse to $\mathscr{F}$. If $\alpha$ is such a one-simplex running from $p_{1}$ in $L_{1}$ to $p_{2}$ in $L_{2}$, we choose an oriented one-simplex $\beta_{2}$ in $L_{2}$ running from $p_{2}$ to $x\left(L_{2}\right)$ and another $\beta_{1}$ in $L_{1}$ running from $x\left(L_{1}\right)$ to $p_{2}$. Now let $\gamma$ be the oriented one-simplex in $I$ running from $x\left(L_{2}\right)$ to $x\left(L_{1}\right)$ and let $w$ be the cycle in $M$ equal to $\alpha+\beta_{2}+\gamma+\beta_{1}$. We define $c(\alpha) \in H_{1}(M) / K$ to be the coset of $[w]$. We made choices for $\beta_{1}$ and $\beta_{2}$, but the homology class [w] is well defined up to an element of $K$. It is immediate that $c$ is holonomy invariant and clear that if a simplex $\alpha$ is subdivided into $\alpha_{1}+\alpha_{2}$, then $c(\alpha)=c\left(\alpha_{1}\right)+$ $c\left(\alpha_{2}\right)$.

Finally if $z=\sum \alpha_{i}$ is a cycle then

$$
\begin{aligned}
\sum\left(\alpha_{i}+\beta_{i 2}+\gamma_{i}+\beta_{i 1}\right)= & \sum \alpha_{i}+\text { cycles in a finite set of leaves } \\
& + \text { a cycle in } I .
\end{aligned}
$$

So, since any cycle in $I$ represents a trivial homology class,

$$
\begin{aligned}
c(z) & =\left[\sum\left(\alpha_{i}+\beta_{i 2}+\gamma_{i}+\beta_{i 1}\right)\right] \bmod K \\
& =\left[\sum \alpha_{i}\right] \bmod K=[z] \bmod K .
\end{aligned}
$$

This completes the proof.

Proof of Corollary. If $H_{1}(L) \rightarrow H_{1}(M)$ is trivial for all $L$, then $K=0$, so $c$ takes values in $H_{1}(M)$. By the universal coefficient theorem,

$$
H^{1}(M ; G) \cong \operatorname{Hom}\left(H_{1}(M ; Z), G\right)
$$

hence any element of $H^{1}(M ; G)$ can be represented by the holonomy invariant cochain $\psi \circ c$ for some $\psi \in \operatorname{Hom}\left(H_{1}(M), G\right)$. This proves the Corollary.

EXAmple. We now give an example of a foliation (due to $M$. Hirsch [1]) in which every leaf has exponential growth and construct a natural holonomy invariant cochain which gives a nonzero cohomology class with coefficients in 
a finite group. By a result of Plante [3], this foliation has no transverse invariant measures.

Let $T=S^{1} \times D^{2}=\left\{(t, \nu)\left|t \in R / Z, \nu \in R^{2},\right| \nu \mid \leqslant 1\right\}$, and choose a smooth embedding $f: T \rightarrow$ int $T$ of the form $f(t, \nu)=\left(p t, f_{1}(t, \nu)\right)$ where $p>2$ is an integer. Now let $\bar{M}=\operatorname{cl}(T-f(T))$ and form a three manifold $M$ by identifying the two boundary components of $\bar{M}$ by the map $f$ (i.e. $(t, v)$ with $|\nu|=1$ is identified with $f(t, \nu))$. Let $\pi: \vec{M} \rightarrow M$ be the natural projection; then there is a unique smooth foliation of $M$ such that for each fixed $t_{0}$, $\pi\left\{\left(t_{0}, \nu\right) \in \bar{M}\right\}$ is contained in a leaf. Thus if $\left(t_{1}, \nu_{1}\right),\left(t_{2}, \nu_{2}\right) \in \bar{M}$, then $\pi\left(t_{1}, \nu_{1}\right)$ and $\pi\left(t_{2}, \nu_{2}\right)$ will lie in the same leaf if and only if $p^{n} t_{1}=p^{m} t_{2}$ in $R / Z$ for some $n$ and $m$.

Each leaf of this foliation is dense and has exponential growth (see [1]). There exists a closed transversal to this foliation which intersects every leaf. It can be given by any smooth embedding $\varphi: S^{1} \rightarrow T$ of the form $\varphi(t)=(t$, $h(t))$ whose image misses $f(T)$. We now let $C=\varphi\left(S^{1}\right)$ be parametrized by $t \in R / Z$ and note that to define a holonomy invariant one cochain it suffices to define a cochain $c$ on $C$ with the property that $c(\sigma)=c(h \circ \sigma)$ where $h$ : $C \rightarrow C$ is given by $h(t)=p t(\bmod Z)$. This is because any sufficiently small transversal to $\mathscr{F}$ is mapped by a holonomy transformation to a subset of $C$ and the holonomy pseudogroup on subsets of $C$ is generated by restrictions of $h$.

We define the desired cochain on $M$ only for the sufficient class of transverse one simplices with vertices at points of the form $\pi(t, \nu)$ where $t=a /(p-1)^{n}(\bmod Z)$ for some integer $a$. To define $c$ on $C$ we define $\bar{c}$ on the universal cover $R$ in such a way that $\bar{c}(p \sigma)=\bar{c}(\sigma)$ and $\bar{c}(\sigma+m)=\bar{c}(\sigma)$ for any integer $m$ and any simplex

$$
\sigma=\left[a /(p-1)^{n}, b /(p-1)^{n}\right] .
$$

The definition is $\bar{c}(\sigma)=(b-a) \bmod (p-1)$ which we consider an element of $Z /(p-1) Z$, and which clearly has the desired properties.

Thus $c$ is defined on simplices in $C$ by lifting them to $R$ and applying $\bar{c}$. It is clear that $c$ extends to a holonomy invariant cochain on $M$. The cocycle it determines gives the value 1 when applied to $C$ (assuming $p>2$ ) and, hence, represents a nonzero cohomology class with coefficients in $Z /(p-1) Z$.

\section{REFERENCES}

1. M. Hirsch, A stable analytic foliation with only exceptional minimal sets, Dynamical Systems-Warwick 1974, Lecture Notes in Math., vol. 468, Springer-Verlag, Berlin and New York, 1975, pp. 9-10.

2. M. Hirsch and W. Thurston, Foliated bundles, invariant measures, and flat manifolds, Ann. of Math. (2) 101 (1975), 369-390. MR 51 \#6842.

3. J. Plante, Foliations with measure preserving holonomy, Ann. of Math. (2) 102 (1975), 327-361.

4. D. Ruelle and D. Sullivan, Currents, flows and diffeomorphisms, Topology 14 (1975), 329-338.

5. W. Thurston, The theory of foliations of codimension greater than one, Comment. Math. Helv. 49 (1974), 214-231. MR 51 \#6846.

Department of Mathematics, Northwestern University, Evanston, Illinois 60201 